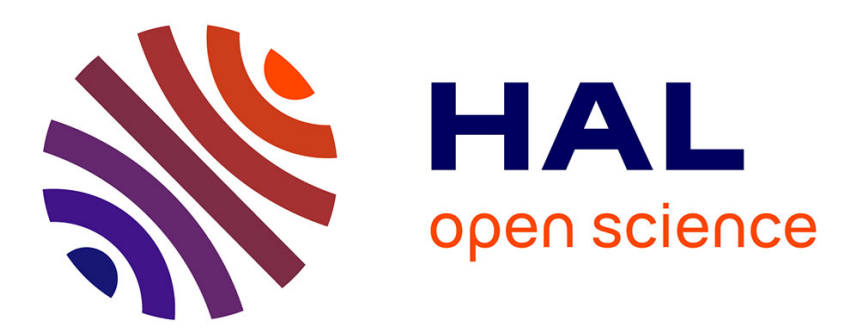

\title{
A computational approach to handle complex microstructure geometries
}

Nicolas Moës, Mathieu Cloirec, Patrice Cartraud, Jean-François Remacle

\section{To cite this version:}

Nicolas Moës, Mathieu Cloirec, Patrice Cartraud, Jean-François Remacle. A computational approach to handle complex microstructure geometries. Computer Methods in Applied Mechanics and Engineering, 2003, 192 (28-30), pp.3163-3177. 10.1016/S0045-7825(03)00346-3 . hal-01389078

\section{HAL Id: hal-01389078 \\ https://hal.science/hal-01389078}

Submitted on 27 Oct 2016

HAL is a multi-disciplinary open access archive for the deposit and dissemination of scientific research documents, whether they are published or not. The documents may come from teaching and research institutions in France or abroad, or from public or private research centers.
L'archive ouverte pluridisciplinaire HAL, est destinée au dépôt et à la diffusion de documents scientifiques de niveau recherche, publiés ou non, émanant des établissements d'enseignement et de recherche français ou étrangers, des laboratoires publics ou privés.

\section{다)(1) $(5$}

Distributed under a Creative Commons Attribution - NonCommercial| 4.0 International 


\title{
A computational approach to handle complex microstructure geometries
}

\author{
N. Moës, M. Cloirec, P. Cartraud ${ }^{\mathrm{a}}$ J.-F. Remacle ${ }^{\mathrm{b}}$ \\ ${ }^{a}$ Laboratoire de mécanique et matériaux, Ecole centrale de Nantes \\ 1 Rue de la Noe, 44321 Nantes, FRANCE \\ ${ }^{\mathrm{b}}$ Université Catholique de Louvain, Département de Génie Civil et Environnement, \\ Bâtiment Vinci, Place du Levant 1, 1348 Louvain-la-Neuve, BELGIUM
}

\begin{abstract}
In multiscale analysis of components, there is usually a need to solve microstructures with complex geometries. In this paper, we use the extended finite element method (X-FEM) to solve scales involving complex geometries. The X-FEM allows one to use meshes not necessarily matching the physical surface of the problem while retaining the accuracy of the classical finite element approach. For material interfaces, this is achieved by introducing a new enrichment strategy. Although the mesh does not need to conform to the physical surfaces, it needs to be fine enough to capture the geometry of these surfaces. A simple algorithm is described to adaptively refine the mesh to meet this geometrical requirement. Numerical experiments on the periodic homogenization of two-phase complex cells demonstrate the accuracy and simplicity of the X-FEM.
\end{abstract}

Key words: Homogenization, surface of discontinuity, X-FEM, periodicity

\section{INTRODUCTION}

The eXtended Finite Element Method simplifies greatly the analysis of structures with complex geometry since the mesh is not required to match this geometry. This property is particularly useful when homogenizing the behavior of micro-structures, complex by nature. We focus here our attention to the application of X-FEM to micro-scale problems involved in the homogenization theory. The homogenization theory is a powerful mean for analyzing and modeling heterogeneous structures. Moreover, in the case of periodic linear media, this theory can be rigorously justified, through convergence results, see e.g. [3], [22]. Thus, as the ratio of the period length to the structure length 
goes to zero, it can be shown that the actual solution of the heterogeneous structure tends to the solution of a problem written for a structure with an homogenized behavior. Furthermore, it turns out that the effective properties are obtained from the solution of a boundary value problem to be solved on a period of the structure, which will be called the basic cell problem in the sequel.

Since the basic cell problem is a boundary value problem, classical numerical methods can be used for computing their solution, see [4] for a state of the art in this matter.

The majority of studies have been based on the Finite Element Method (FEM), see [8], [12] and references herein, and only few papers present a Boundary Element Method implementation, see e.g. [10], [18]. Let us also mention the Fast Fourier Transforms based numerical method [17]. This method is well suited for periodic homogenization, and is very efficient compared to FEM, especially in the linear range [12], provided that no voids or rigid inclusions are present in the basic cell.

The advantages of the FEM in micromechanical analysis are indeed the same as in standard engineering problems: its flexibility, and its applicability to nonlinear problems, anisotropic materials, and arbitrary geometries. Following [4], one may distinguish, in FEM based periodic homogenization studies, four main groups.

In most of the studies, the mesh models the heterogeneities boundaries in the unit cell, i.e. only one phase is present within each finite element. The main drawback of such an approach is the explicit microstructure modeling, which leads to problems for generating the mesh for complex geometries, and requires the use of sophisticated tools, see e.g. [26].

One way to overcome these difficulties is to use a digital image based FEM technique, as initially proposed in [9]. It consists in using a uniform mesh that has the same resolution as the digital image, and then to identify each pixel or voxel as a finite element. Such an approach, however, leads to models which are computationally expensive.

A third approach also uses regular meshes, but the interfaces between constituents may be represented independently of element boundaries. For an element cut by an interface, the heterogeneity is treated at the integration point level [27]. As we shall see in the numerical experiments, this strategy yields a reasonable rate of convergence for the homogenized parameters of the basic cell but suffers a slow rate of convergence for the quality of the overall stress distribution over the basic cell (energy norm in the stresses).

Another technique is the Voronoi cell finite element model [7], in which an 
element incorporates the effect of an embedded inclusion in the matrix. Both stress and displacement fields need to be discretized with this technique.

The eXtended Finite Element Method provides yet another way to solve the basic cell problem. The advantage of this approach is to retain all the advantages of the finite element approach (applicability to nonlinear and anisotropic constitutive laws, wide range of codes already written, robustness, ...) while considerably easing the meshing step. Indeed, the physical surfaces of the problem do not need to be meshed. They are taken into account by enriching the finite element approximation space through the partition of unity technique [11]. The adequate choice of the enrichment function for cracks was discussed in $[1,14]$, for intersecting cracks and voids in [6]. Concerning material interfaces, an enrichment was introduced in [24]. This latter paper was also the first one to use a level set representation of surfaces in the X-FEM context. This representation not only reduces the surface storage to a usual finite element field but also provides a natural way to express the enrichment. Note that in order to build the level set representation of the material interfaces, the CAD geometries of these surfaces are not necessarily needed. All that is needed is a function returning for one $(\mathrm{x}, \mathrm{y}, \mathrm{z})$ point the distance to the closest material interface. In other words, the X-FEM approach coupled to the level set representation of the surfaces not only simplifies the mesh step but also the geometrical data pre-processing. In this paper, we improve the enrichment functions for material interfaces and obtain a convergence rate very close to the one obtained with regular finite elements (i.e. conforming meshes).

The remainder of this paper is organized as follows. The basic cell problem is described in Section 2. The X-FEM approach to solve the basic cell problem is detailed in Section 3 and a new enrichment function for material interface is introduced and compared to previous approaches. The fourth section uses the X-FEM to homogenize three different basic cells: a cell with orthogonal non-woven fibers, a cell with woven fiber and, finally, a cell with randomly placed spherical inclusions.

\section{THE BASIC CELL PROBLEM}

The formulation of the basic cell problem for composites with periodic microstructure can be derived in a systematic way using the two-scale asymptotic expansion method [3], [22], or following a process which is also valid for random media [25], [12]. The latter approach is used in this paper.

The microstructure description is achieved through the definition of a representative volume element, containing all the geometrical and material data. In the case of a periodic medium, the representative volume element is simply 
a basic cell $\Omega$, which forms the composite by spatial repetition.

At the macroscopic scale, stress and strain fields are denoted by $\boldsymbol{\Sigma}$ and $\boldsymbol{E}$, which are the average of the corresponding microscopic field, $\boldsymbol{\sigma}$ and $\boldsymbol{e}$, on the basic cell (the macroscopic and microscopic scales are denoted by $\boldsymbol{x}$ and $\boldsymbol{y}$, respectively):

$$
\left\{\begin{array}{l}
\boldsymbol{\Sigma}(\boldsymbol{x})=<\boldsymbol{\sigma}(\boldsymbol{x}, \boldsymbol{y})>=\frac{1}{|\Omega|} \int_{\Omega} \boldsymbol{\sigma}(\boldsymbol{x}, \boldsymbol{y}) d \Omega \\
\boldsymbol{E}(\boldsymbol{x})=<\boldsymbol{e}(\boldsymbol{x}, \boldsymbol{y})>
\end{array}\right.
$$

The homogenized behavior relating $\boldsymbol{\Sigma}$ to $\boldsymbol{E}$ is built using a localization process which consists in submitting the composite structure to a given macroscopic stress or strain state. In the case of a periodic microstructure, such a problem can be studied on a single basic cell, thanks to periodic boundary conditions. Moreover, the local periodicity of the strain $\boldsymbol{e}(\boldsymbol{x}, \boldsymbol{y})$ with respect to the microscopic scale $\boldsymbol{y}$, and the relation $\boldsymbol{E}(\boldsymbol{x})=<\boldsymbol{e}(\boldsymbol{u}(\boldsymbol{x}, \boldsymbol{y}))>$ leads to the following decomposition of the displacement field:

$$
\boldsymbol{u}(\boldsymbol{x}, \boldsymbol{y})=\boldsymbol{E}(\boldsymbol{x}) \cdot \boldsymbol{y}+\boldsymbol{u}^{m}(\boldsymbol{y}), \boldsymbol{u}^{m} \quad \text { periodic on } \partial \Omega
$$

Therefore, with the data $\boldsymbol{E}$, the basic cell (or localization) problem is defined by:

Find $\boldsymbol{\sigma}, \boldsymbol{e}, \boldsymbol{u}^{\boldsymbol{m}}$ such that:

$$
\left\{\begin{array}{l}
\operatorname{div} \boldsymbol{\sigma}(\boldsymbol{y})=\mathbf{0} \\
\boldsymbol{\sigma}(\boldsymbol{y})=\boldsymbol{a}(\boldsymbol{y}): \boldsymbol{e}(\boldsymbol{y})=\boldsymbol{a}(\boldsymbol{y}):\left(\boldsymbol{E}+\boldsymbol{e}\left(\boldsymbol{u}^{\boldsymbol{m}}(\boldsymbol{y})\right)\right) \\
\boldsymbol{u}^{\boldsymbol{m}} \text { periodic } \\
\boldsymbol{\sigma} . \mathbf{n} \text { anti-periodic }
\end{array}\right.
$$

where "periodic" ("anti-periodic") means that values on opposite sides of the boundary $\partial \Omega$ are equal (opposite). The problem (3) admits a unique solution, up to a rigid body translation. Once problem (3) is solved, the strain localization tensor $\boldsymbol{D}$ is obtained

$$
e(\boldsymbol{y})=\boldsymbol{D}(\boldsymbol{y}): \boldsymbol{E}
$$

and the effective stiffness $\boldsymbol{a}^{\text {hom }}$ may be computed as:

$$
\boldsymbol{a}^{\text {hom }}=<\boldsymbol{a}(\boldsymbol{y}): \boldsymbol{D}(\boldsymbol{y})>
$$


For a linear elastic medium, the complete determination of $\boldsymbol{a}^{\text {hom }}$ requires the solution of problem (3) for 6 independent data $\boldsymbol{E}$. One way to proceed is to consider successively the elementary macroscopic strain states corresponding to one component of $\boldsymbol{E}$ equal to unity, the others being zero. Thus, introducing the second order tensor $\boldsymbol{I}^{i j}$ defined by:

$$
\boldsymbol{I}_{k h}^{i j}=\frac{1}{2}\left(\delta_{i k} \delta_{j h}+\delta_{i h} \delta_{j k}\right)
$$

and denoting $\boldsymbol{u}^{\mathbf{m}_{i j}}$ the solution of problem (3) with the data $\boldsymbol{I}^{i j}$, one has:

$$
\boldsymbol{D}_{k h i j}=\left(\boldsymbol{e}\left(\boldsymbol{u}^{\mathbf{m}_{i j}}(\boldsymbol{y})\right)\right)_{k h}
$$

The problem (3) corresponds to the strain approach, the data being the macroscopic strain $\boldsymbol{E}$. One can also use a stress approach, with the data $\boldsymbol{\Sigma}$, thus leading to effective compliance $\boldsymbol{A}^{\text {hom }}$, which is equal to the inverse of $\boldsymbol{a}^{\text {hom }}$.

It is important to mention that, in the framework of periodic homogenization, non periodic materials (i.e. with some randomness in the geometrical shape of the heterogeneities and their spatial distribution) may be studied. In that case, however, one has to use a sufficiently large basic cell, in order to obtain a statistically representative response and accurate effective properties. In this paper, such examples of basic cells will be treated.

The variational formulation of the basic cell problems (3), for a data $\boldsymbol{E}=\boldsymbol{I}^{\boldsymbol{i j}}$ is given by:

$$
\left\{\begin{array}{l}
\text { Find } \boldsymbol{u}^{\mathbf{m}_{i j}} \text { periodic such that } \\
a\left(\boldsymbol{u}^{\mathbf{m}_{i j}}, \boldsymbol{v}\right)=-a\left(\boldsymbol{I}^{\boldsymbol{i j}}, \boldsymbol{v}\right) \forall \boldsymbol{v} \text { periodic }
\end{array}\right.
$$

where we use the bilinear form:

$$
a(\boldsymbol{u}, \boldsymbol{v})=\frac{1}{|\Omega|} \int_{\Omega} \boldsymbol{e}(\boldsymbol{u}): \boldsymbol{a}: \boldsymbol{e}(\boldsymbol{v}) d \Omega
$$

The effective properties are determined from:

$$
a_{i j k l}^{h o m}=a\left(\boldsymbol{I}^{k \boldsymbol{l}}+\boldsymbol{u}^{\mathbf{m}_{k l}}, \boldsymbol{I}^{\boldsymbol{i j}}+\boldsymbol{u}^{\mathbf{m}_{i j}}\right)=a\left(\boldsymbol{I}^{k \boldsymbol{l}}, \boldsymbol{I}^{\boldsymbol{i j}}\right)-a\left(\boldsymbol{u}^{\mathbf{m}_{k l}}, \boldsymbol{u}^{\mathbf{m}_{i j}}\right)
$$


Since in this paper a displacement approach is used, the right-hand side of (8) is known. Therefore, the only specific feature of problem (8) is the periodicity condition [12].

\section{X-FEM DISCRETIZATION}

In this paper, we consider the homogenization of two-phase basic cells. The material interface separating the two phases is described by a level set function. The level set representation is then used to express a jump in the strain field within the elements crossed by the material interface.

\subsection{Level set representation}

Consider a two-phase basic cell. The two phases are separated by an interface which we may locate either explicitely (set of CAD entities) or implicitely by assigning to each node $I$ of the mesh, the distance $\phi_{I}$ to the interface (with a positive sign if node $I$ is located in one phase and a negative sign if node $I$ is located in the other phase). Next, we may interpolate these nodal informations using the finite element shape functions, $N_{I}(\mathbf{x})$, yielding the level set expression:

$$
\phi(\mathbf{x})=\sum_{\mathbf{I}} \phi_{\mathbf{I}} \mathbf{N}_{\mathbf{I}}(\mathbf{x})
$$

where the sum over $I$ indicates a sum over the nodes of the mesh (more precisely only the nodes belonging to the element containing point $\mathbf{x}$ ). The isozero of the level set function $\phi$ approximates the true location of the interface. A strategy for constructing the level set function in the case where the interface is known only by a set of points is described in [2].

With the eXtended Finite Element Method, the mesh does not need to conform to the interface but the mesh has to be fine enough to locate precisely the interface and to accurately resolve the displacement field i.e. two kinds of error appear: geometrical and numerical errors. Since a level set interpolated on the finite element mesh is used to locate the interface, one can notice that both geometrical and numerical errors are reduced as the mesh gets finer (as with the FEM). As an example, Figure ?? shows the geometry of the basic cell for a landing helicopter grid located on the deck of an aircraft carrier. Using the coarse mesh, Figure 2 (left), the iso-zero level set obtained is shown Figure 2 (right). It can be seen that the geometrical representation is poor. A finer mesh, Figure 3 (left), gives a much better representation of the surface location, Figure 3 (right). 
The finer mesh was obtained using a simple adaptive mesh refinement strategy. The idea is to reduce the size of the elements located close to the physical surface if the local radius of curvature of the surface is smaller than the characteristic element size. The radius of curvature $\rho$ on an element is computed by the second gradient of the level set interpolation over the element $[23,15]$ and the characteristic element size $l_{c}$ is computed by $l_{\mathrm{c}}=(V * d !)^{1 / d}$ where $V$ is the element volume and $d$ the problem dimension. An element is tagged for refinement if

$$
l_{c}>\alpha \rho \text { and }|\bar{\phi}|<\beta l_{\text {tot }}
$$

where $\bar{\phi}$ is the level set value at the centroid of the element and $l_{t o t}$ the side length of the basic cell. The dimensionless parameters $\alpha$ and $\beta$ govern the extent and level of refinement. Based on the elements tagged for refinement, the mesh can be refined where needed. In order to meet the condition $l_{c}>\alpha \rho$ starting from one initial coarse mesh, several intermediary meshes are usually needed. As meshes become finer, the evaluation of the curvature $\rho$ becomes more accurate. The process ends when no element is tagged.

The mesh shown Figure 3 was obtained with $\alpha=0.1$ and $\beta=0.03$. The gmsh meshing tool [19] and the aomd mesh database library [20,21] were used to refine the mesh based on the elements tagged.

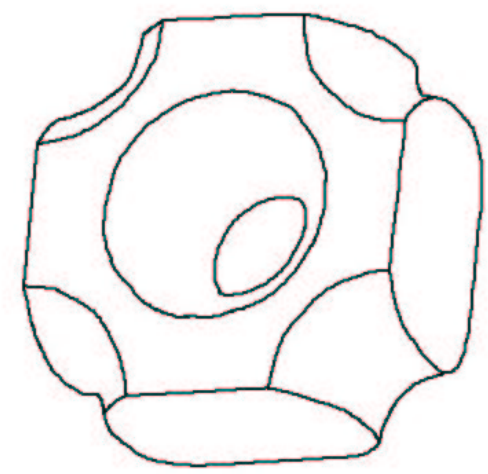

Fig. 1. Unit cell geometry for an helicopter landing grid.

\subsection{Enrichment strategy}

Once the level set function is defined, we can express the X-FEM approximation. The classical finite approximation over the basic cell is given by

$$
\mathbf{u}^{\mathrm{FEM}}=\sum_{\mathbf{I}} \mathbf{u}_{\mathbf{I}} \mathbf{N}_{\mathbf{I}}(\mathbf{x})
$$



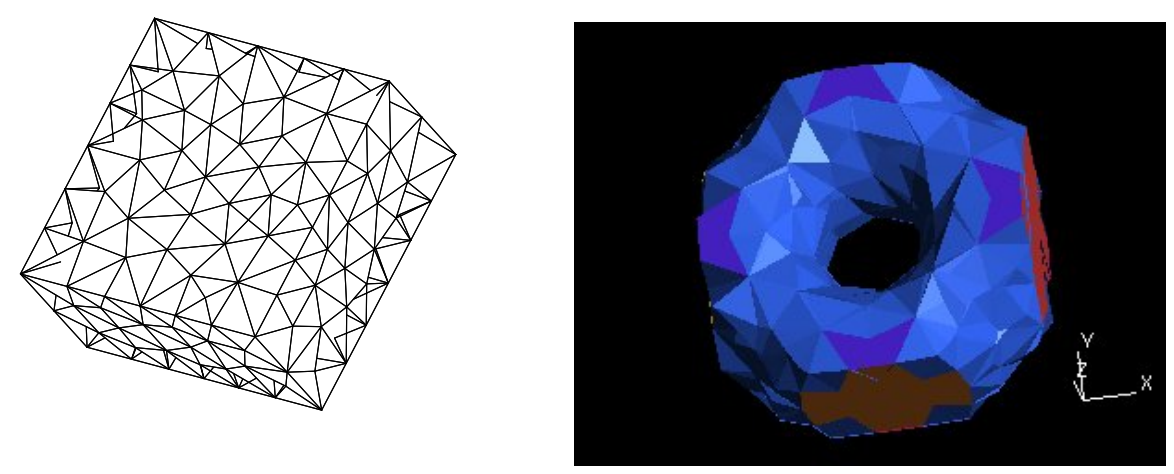

Fig. 2. A coarse mesh and the corresponding iso-zero level set.
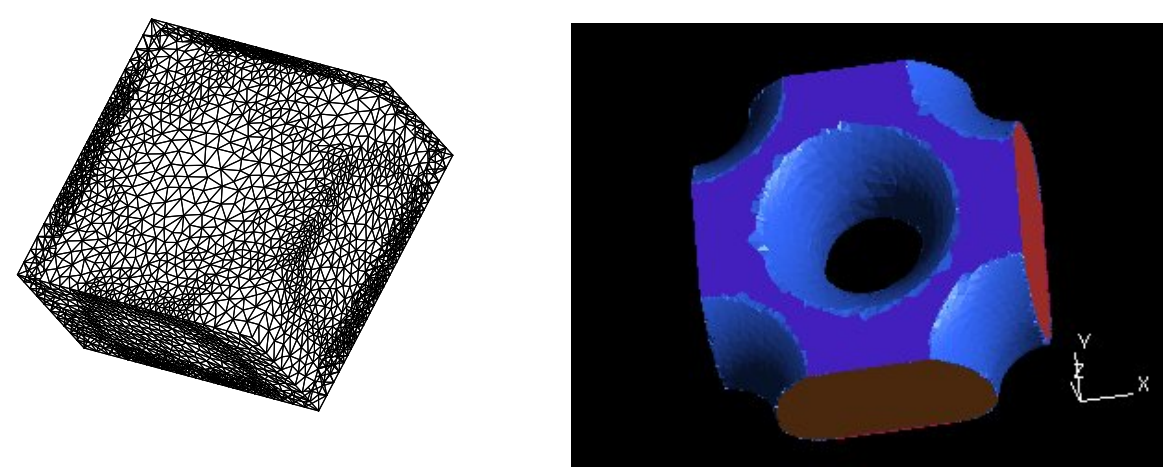

Fig. 3. A refined mesh and the corresponding iso-zero level set.

If the finite element mesh conforms to the material interface, the (linear) approximation above yields an $O(h)$ convergence rate in the energy norm (provided the solution is smooth) [8]. On the contrary, as will be shown in the numerical experiments, if the finite element does not conform to the interface, a poor rate of convergence is obtained. For instance, a two-phase 1D problem with a mesh non-conforming to the interfaces gives an asymptotic rate of convergence as poor as $O(\sqrt{h})$ [16]. In order to avoid poor rates of convergence, the X-FEM adds an enrichment to the classical finite element approximation through a partition of unity technique [11]:

$$
\mathbf{u}^{\mathrm{X}-\mathrm{FEM}}=\sum_{\mathbf{I}} \mathbf{u}_{\mathbf{I}} \mathbf{N}_{\mathbf{I}}(\mathbf{x})+\mathbf{u}^{\mathrm{enr}}, \quad \mathbf{u}^{\mathrm{enr}}=\sum_{\mathbf{J}} \mathbf{a}_{\mathbf{J}} \mathbf{N}_{\mathbf{J}}(\mathbf{x}) \mathbf{F}(\mathbf{x})
$$

The additional degrees of freedom $\mathbf{a}_{\mathbf{J}}$ are added at the nodes for which the support is split by the interface. The function $F$ is called the enrichment function. It is discontinuous in its derivative across the interface. In [24], the enrichment is defined as the absolute value of the level set function which indeed has a discontinuous first derivative on the interface:

$$
F^{1}(\mathbf{x})=\left|\sum_{\mathbf{I}} \phi_{\mathbf{I}} \mathbf{N}_{\mathbf{I}}(\mathbf{x})\right|
$$


A similar choice[2], is to define $\mathbf{u}^{\mathbf{e n r}}$ as

$$
\mathbf{u}^{\mathrm{enr}}=\sum_{\mathbf{J}} \mathbf{a}_{\mathbf{J}} \mathbf{N}_{\mathbf{J}}(\mathbf{x})\left(\mathbf{F}^{\mathbf{1}}(\mathbf{x})-\mathbf{F}^{\mathbf{1}}\left(\mathbf{x}_{\mathbf{J}}\right)\right)
$$

A closer look at the two enrichments above indicates that they will yield the same approximation space. However, the conditioning number of the two resulting matrices will be different in general. It was shown in [24] and [2] that a smooting of $F^{1}$ away from the element layer containing the interface somewhat improved the convergence. The effect of this smoothing in $1 \mathrm{D}$ is illustrated Figure 4. For 2D problems, the smoothing strategy is detailed in [24].

In this paper, we suggest another choice for the enrichment function:

$$
F^{2}(\mathbf{x})=\sum_{\mathbf{I}}\left|\phi_{\mathbf{I}}\right| \mathbf{N}_{\mathbf{I}}(\mathbf{x})-\left|\sum_{\mathbf{I}} \phi_{\mathbf{I}} \mathbf{N}_{\mathbf{I}}(\mathbf{x})\right|
$$

This enrichment is shown Figure 4 in the 1D case. For two- and three-dimensional problems, the enrichment function $F^{2}$ is a ridge centered on the interface and has zero value on the elements which are not crossed by the interface.

Remark: If the mesh conforms to the material interface, no nodes are enriched since no element is cut by the material interface. The X-FEM then does behave as the FEM. On an element cut by the interface, the integration needs to be performed carefully. First, we divide the element into subdomains matching the material interface as described in [14]. Then, the number of integration points over each subdomain is chosen so that the integration is "exact" (note that $F(x) N_{J}(x)$ is polynomial). On the elements, not cut by the interface but neighbouring an element cut (for instance element A, Figure 4), no subdivision needs to be performed but the order of the integration needs to be chosen with care since the enrichment acts on the element.

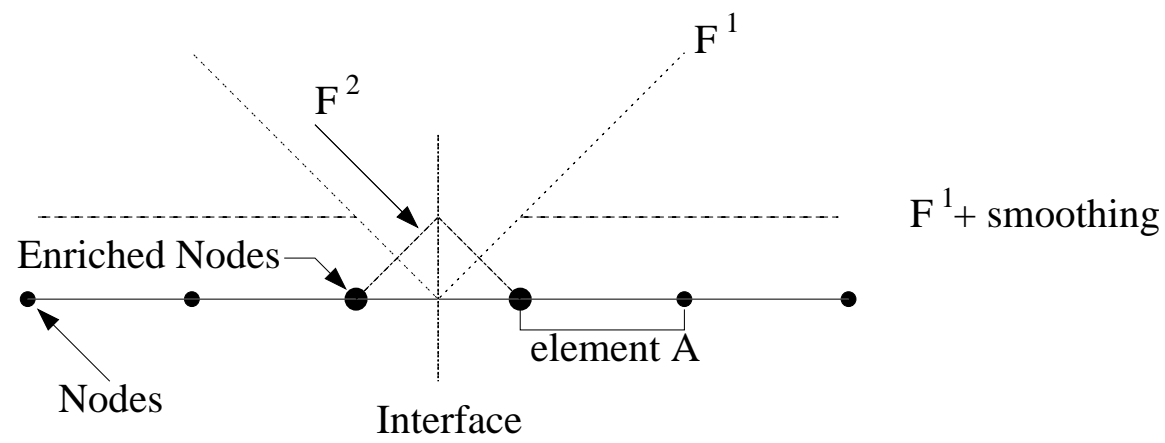

Fig. 4. Several choices for the enrichment function. 


\subsection{Convergence tests}

In order to compare the convergence rate of the classical and extended finite element method, we consider a 2D circular and a 3D spherical inclusion under tension in an infinite domain. These benchmark problems were already considered in [24] and [2] in 2D and 3D, respectively. In order to compare the numerical and exact solution, we use the energy norm. Let $u^{h}$ be an approximate solution and $u^{e x}$ the exact solution, the "exact" error is defined by:

$$
\varepsilon=\frac{\sqrt{\int_{\Omega} e\left(u^{h}-u^{e x}\right): a: e\left(u^{h}-u^{e x}\right) d \Omega}}{\sqrt{\int_{\Omega} e\left(u^{e x}\right): a: e\left(u^{e x}\right) d \Omega}}
$$

Concerning the material properties, the Poisson ratio is set to 0.25 for the inclusion and to 0.3 for the matrix. The Young modulus ratio between the inclusion and matrix is set to 10. Figures 5 and 6 show the decrease in the energy error with respect to the exact solution in four different cases:

- A finite element mesh conforming to the material interface (denoted FEM);

- A finite element computation with a non conforming mesh (denoted FEMnon-conforming). On the elements containing the material interface, the integration of the bilinear form is performed using the appropriate constitutive law at each integration point.

- The extended finite element method using the enrichment $F^{1}$ improved by a smoothing (denoted X-FEM-1 + smoothing);

- The extended finite element method using the new enrichment, $F^{2}$, given equation (17) (denoted X-FEM-2).

It can be seen that the new enrichment X-FEM-2 improves somewhat the previous enrichment strategy X-FEM-1. The X-FEM convergence rate is now very close to the "optimal" finite element convergence. We also note, as expected, the poor convergence rate of the non-conforming FEM approach.

Although, the error norm used is a global norm, most of the error contribution comes from the element cut by the interface as can be seen in Figure 7 showing the contribution to the error of the disc (or sphere), D(r), of radius $r$.

$$
\varepsilon(r)=\frac{\sqrt{\int_{D(r)} e\left(u^{h}-u^{e x}\right): a: e\left(u^{h}-u^{e x}\right) d \Omega}}{\sqrt{\int_{\Omega} e\left(u^{e x}\right): a: e\left(u^{e x}\right) d \Omega}}
$$

The error norm thus depends directly on the quality of stresses along the 


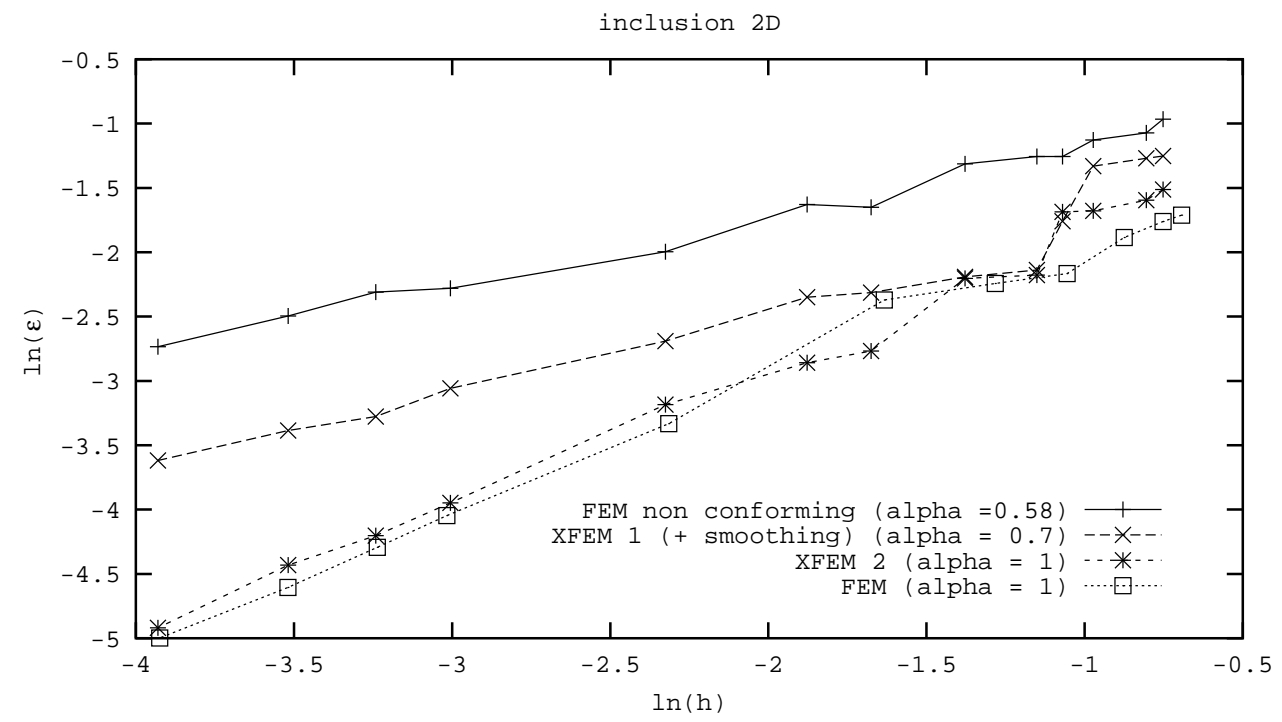

Fig. 5. Convergence rates for a 2D circular inclusion problem. The alpha value indicates the rate of convergence with respect to the average element size $\mathrm{h}$.

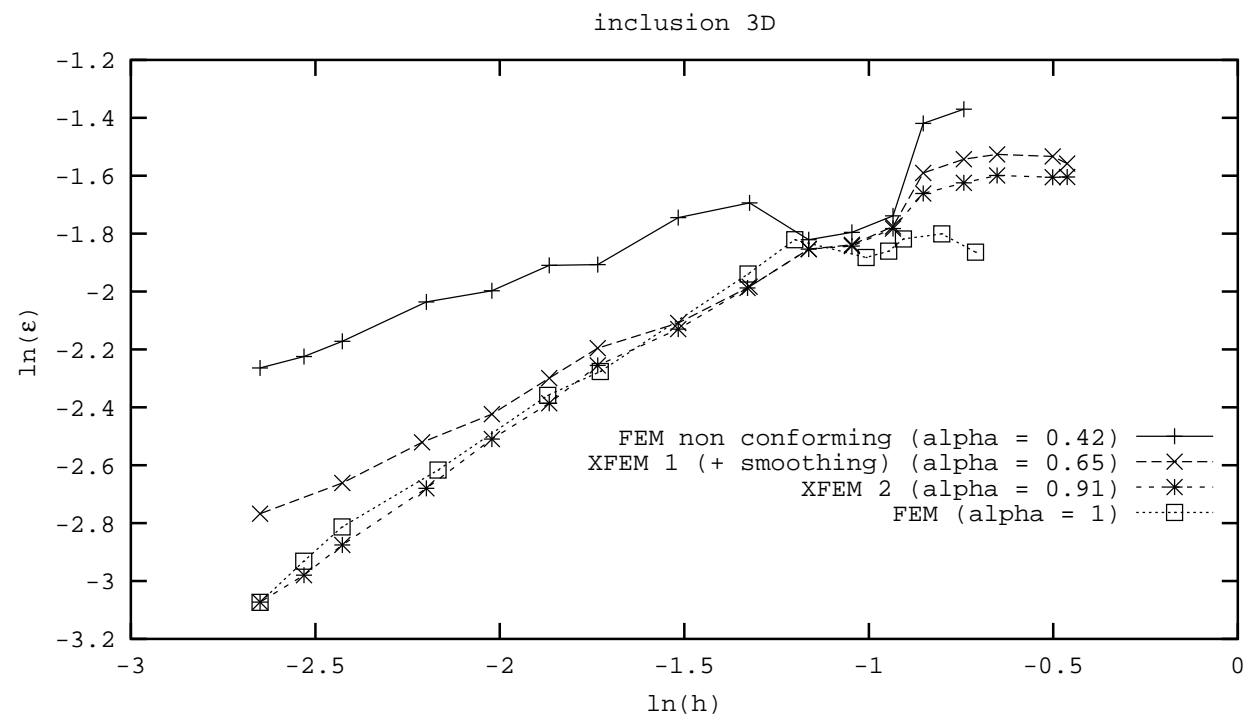

Fig. 6. Convergence rates for a $3 \mathrm{D}$ spherical inclusion problem. The alpha value indicates the rate of convergence with respect to the average element size $\mathrm{h}$.

material interface.

\subsection{Periodicity conditions}

To take into account the periodicity condition, we use meshes which are periodic. The displacement constraints on the faces of the unit cell are condensed out of the stiffness matrix at the assembly stage. We mentioned earlier that a 

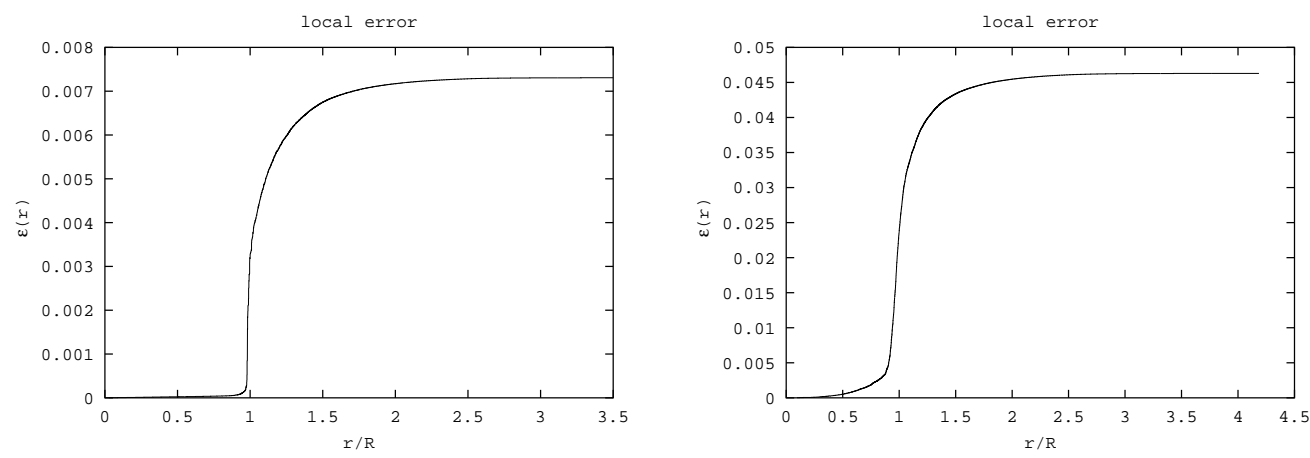

Fig. 7. Error contribution $\varepsilon(r)$ with respect to $\mathrm{r} / \mathrm{R}$ for the $2 \mathrm{D}$ circular inclusion problem (left) and the $3 \mathrm{D}$ spherical inclusion problem (right). In the both cases, $\mathrm{R}$ is the radius of the inclusion.

node is enriched if its support is crossed by the interface. The support must take into account the periodicity of the mesh. For instance, Figure 8, the support of node $K$ is based on the four shaded elements. Thus node $K$ will be enriched since its support is crossed by the material interface. Nodes $K$ and $L$ have the same number of degrees of freedom with the same values.

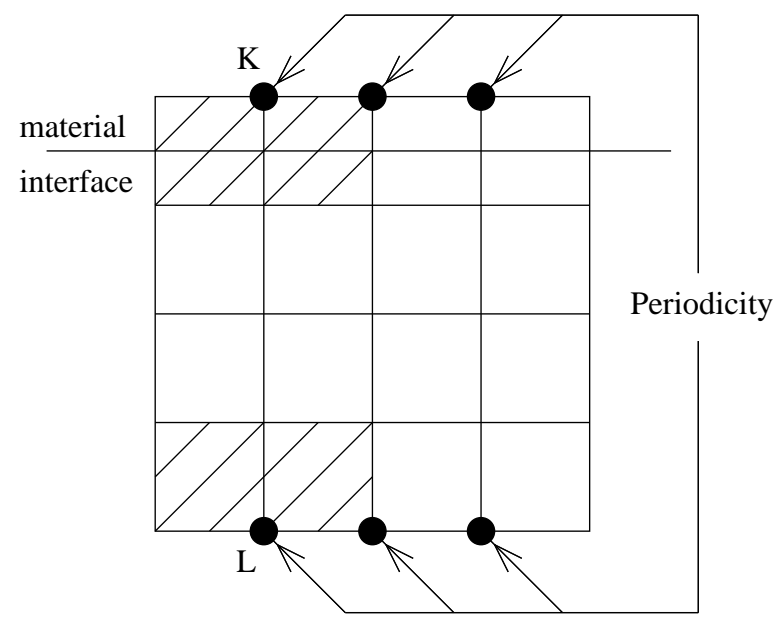

Fig. 8. The support of a node located on the side of a $2 \mathrm{D}$ cell.

\section{NUMERICAL EXPERIMENTS}

We consider three different cell geometries: an non-woven composite cell with orthogonal fibers, a woven composite cell and a randomly filled cell with spherical inclusions. In each case, we compare the X-FEM results to existing simulations based on the classical finite element method. 


\section{$4.1 \quad$ Non-woven cell}

This example is taken from [5]. The cell geometry is shown Figure 9. Although several fibers are involved only one level set function is needed. The cell side length is 2 and the fibers radius is 0.45 . The material properties are representative of E-glass fibers and epoxy resin matrix (in GPa): $E_{f}=72.0, G_{f}=$ $27.7, \nu_{f}=0.30, E_{m}=3.5, G_{m}=1.3, \nu_{m}=0.35$. The homogenized stiffness is given Table 1 for the X-FEM and FEM analysis. Figure 10 compares the shear $x y$ mode obtained with the two approaches. It is clear from these plots that the displacement field gradient is indeed discontinuous at the material interface. The X-FEM mesh is a uniform 4-node tetrahedron mesh shown Figure 11 whereas the FEM mesh used for comparison is shown Figure 13. Note that the $\mathrm{X}$-FEM mesh used is composed of many more elements than the FEM mesh. The X-FEM mesh used is uniform as shown Figure 11 whereas the FEM mesh was optimized. Note that the strategy designed in section Section 3.1 could be used to optimize the X-FEM mesh. Figure 9 shows the iso-zero of the level set field.

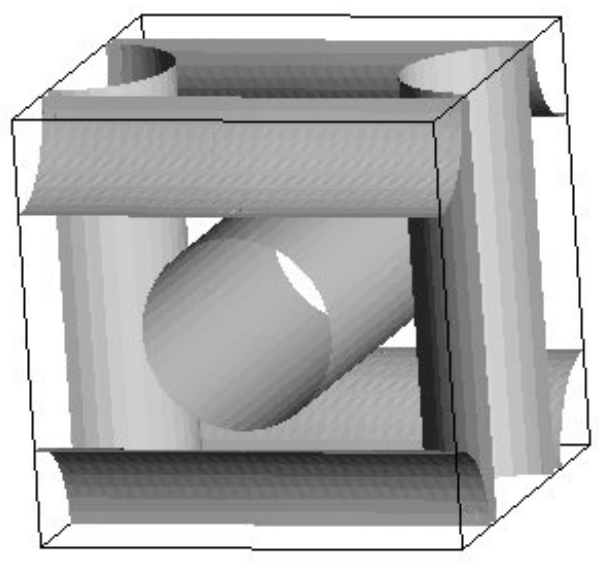

Fig. 9. The unit cell of non-woven composite.

\subsection{Woven cell}

The second example is based on the numerical experiment carried out in [8]. The fibers geometry is defined by a disc following a sinus path. The geometry of the unit cell is shown Figure 12 for "two plies". The finite element mesh designed in [8] is shown Figure 13 (for one ply). For this example, the XFEM mesh used is the same as for the non-woven cell (Figure 11). The boron 


$$
\left[\boldsymbol{a}_{\text {non-woven }}^{\text {hom }}\right]=\left[\begin{array}{cccccc}
21.5 / 21.1 & 5.6 / 5.3 & 5.6 / 5.3 & 0 / 0 & 0 / 0 & 0 / 0 \\
5.6 / 5.3 & 21.5 / 21.1 & 5.6 / 5.3 & 0 / 0 & 0 / 0 & 0 / 0 \\
5.6 / 5.3 & 5.6 / 5.3 & 21.5 / 21.1 & 0 / 0 & 0 / 0 & 0 / 0 \\
0 / 0 & 0 / 0 & 0 / 0 & 3.5 / 3.4 & 0 . / 0 . & 0 / 0 \\
0 / 0 & 0 / 0 & 0 / 0 & 0 / 0 & 3.5 / 3.4 & 0 / 0 \\
0 / 0 & 0 / 0 & 0 / 0 & 0 / 0 & 0 / 0 & 3.5 / 3.4
\end{array}\right]
$$

Table 1

Homogenized behavior for the non-woven composite: X-FEM/FEM results.
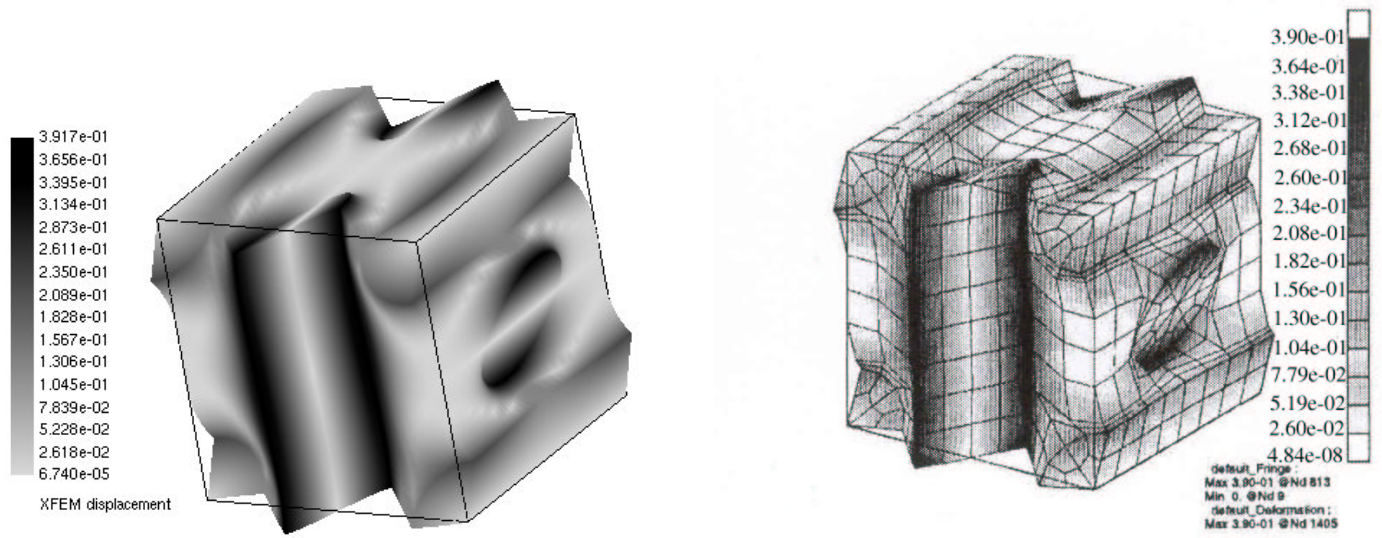

Fig. 10. Shear $x y$ deformation modes obtained by the X-FEM (left) and FEM (right) computations.

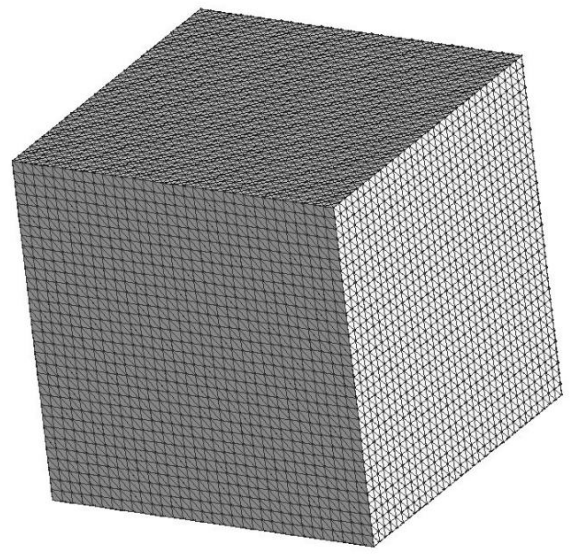

Fig. 11. The uniform mesh used for the X-FEM computations. 


$$
\left[\boldsymbol{a}_{\text {woven }}^{\text {hom }}\right]=\left[\begin{array}{cccccc}
121.2 / 119.2 & 58.2 / 57.8 & 57.4 / 57.0 & 0 / 0 & 0 / 0 & 0 / 0 \\
58.2 / 57.8 & 117.9 / 116.7 & 58.3 / 57.8 & 0 / 0 & 0 / 0 & 0 / 0 \\
57.4 / 57.0 & 58.3 / 57.8 & 121.3 / 119.9 & 0 / 0 & 0 / 0 & 0 / 0 \\
0 / 0 & 0 / 0 & 0 / 0 & 30.6 / 30.3 & 0 / 0 & 0 / 0 \\
0 / 0 & 0 / 0 & 0 / 0 & 0 / 0 & 29.9 / 29.8 & 0 / 0 \\
0 / 0 & 0 / 0 & 0 / 0 & 0 / 0 & 0 / 0 & 30.6 / 30.3
\end{array}\right]
$$

Table 2

Homogenized behavior for the woven composite: X-FEM/FEM results.

fibers and aluminum matrix have the following properties: $E_{f}=400 G P a, \nu_{f}=$ $0.3, E_{m}=72 G P a, \nu_{m}=1 / 3$. The homogenized behavior obtained by the XFEM and FEM computations are given in Table 2 and the shear $x y$ mode obtained with the X-FEM is shown Figure 14.

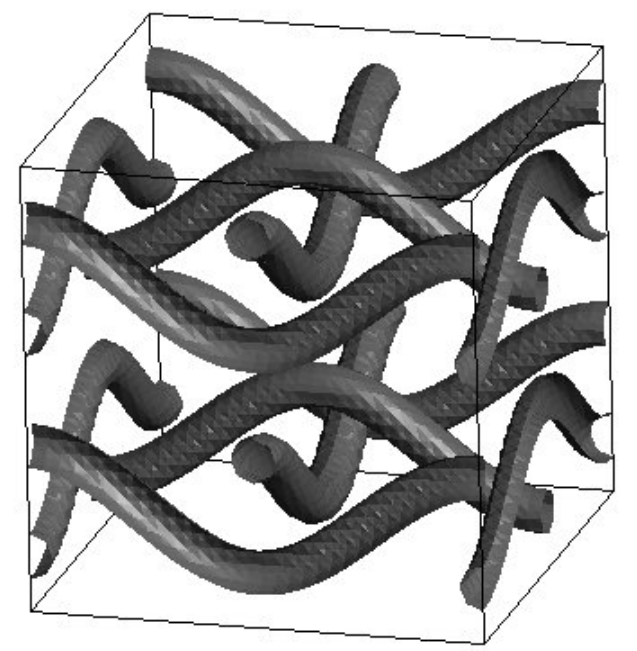

Fig. 12. Woven cell geometry.
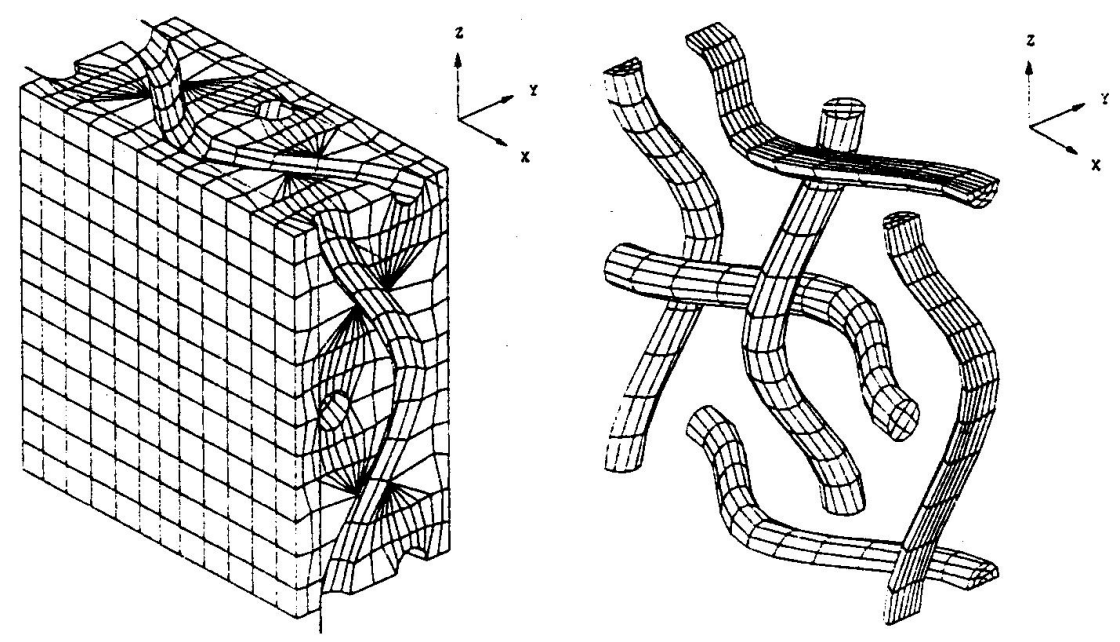

Fig. 13. Mesh designed in the paper by Kikuchi et al. [8] for the FEM analysis of the woven unit cell. 


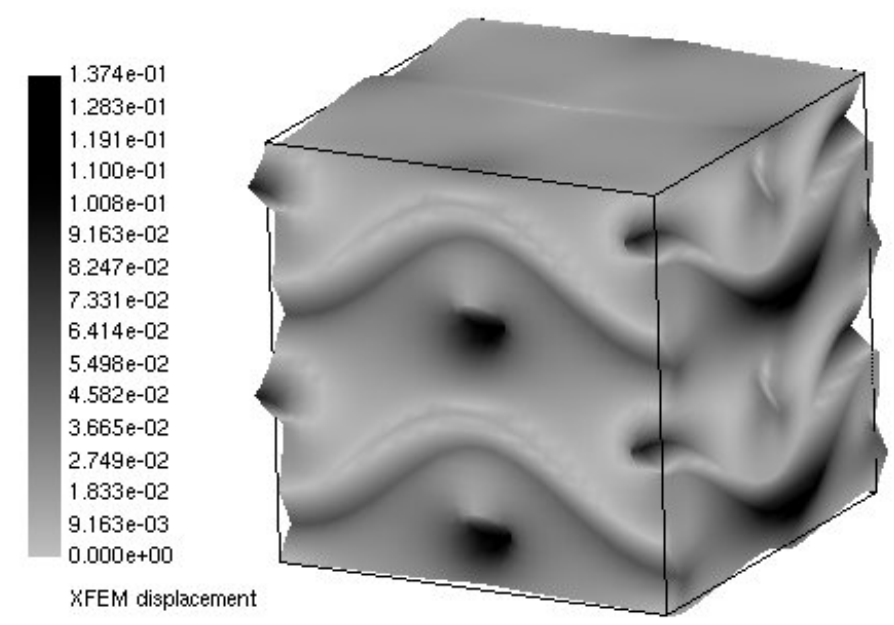

Fig. 14. Shear $x y$ deformation modes obtained by the X-FEM for the woven microstructure.

\subsection{Random cell}

As a last example, we consider a random media filled with spherical inclusions. This example was solved by [13] using a Fast Fourier Transform approach. In order to take into account the randomness of the spatial distribution of the inclusions, many basic cells have to be considered for the computation of the homogenized properties. Therefore, the X-FEM approach is particularly effective since the same mesh can be used for all the simulations (whereas the level set field must be regenerated for each draw). Moreover, it is also applicable to voids (in which case the enrichment is not used and the voids are taken into account using the strategy designed in $[6,2])$.

The Figures 15 and 16 show draws of 8 and 32 particles, respectively. The draws were forced to produce a periodic cell structure. The location of the sphere centers are randomly generated. If a sphere cannot be introduced in the cell because it intersects other spheres already present, a new center location is generated. If a new sphere intersects the cell boundary, its periodic "images" are also introduced.

The particules and matrix properties are $E_{p}=70 G P a, \nu_{p}=0.2$ and $E_{m}=$ $3 G P a, \nu_{m}=0.35$, respectively and the particule volume ratio is set to $26.78 \%$. The X-FEM mesh used is still the uniform mesh shown Figure 11 and the shear $x y$ mode obtained with the X-FEM is shown Figure 17. The homogenized stiffness obtained along the $x$ axis $\left(a_{1111}^{\text {hom }}\right)$ is 7.611 for 8 particles and 7.711 for 32 particles. These results are very close to the ones reported in [13]. The 
latter where computed with many more draws (and thus more reliable): $a_{1111}^{\text {hom }}$ $=7.675$ for 8 particles, 7.664 for 64 particles and 7.667 for 420 particles.
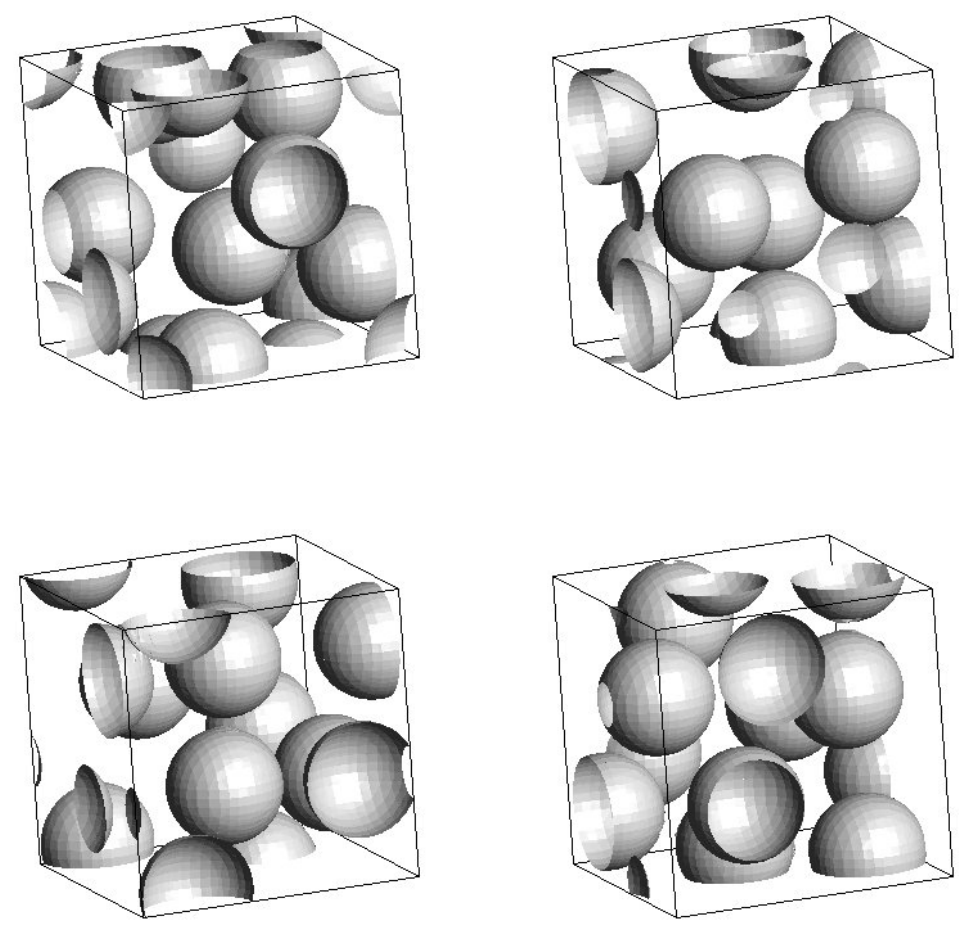

Fig. 15. Four different draws with 8 particles.
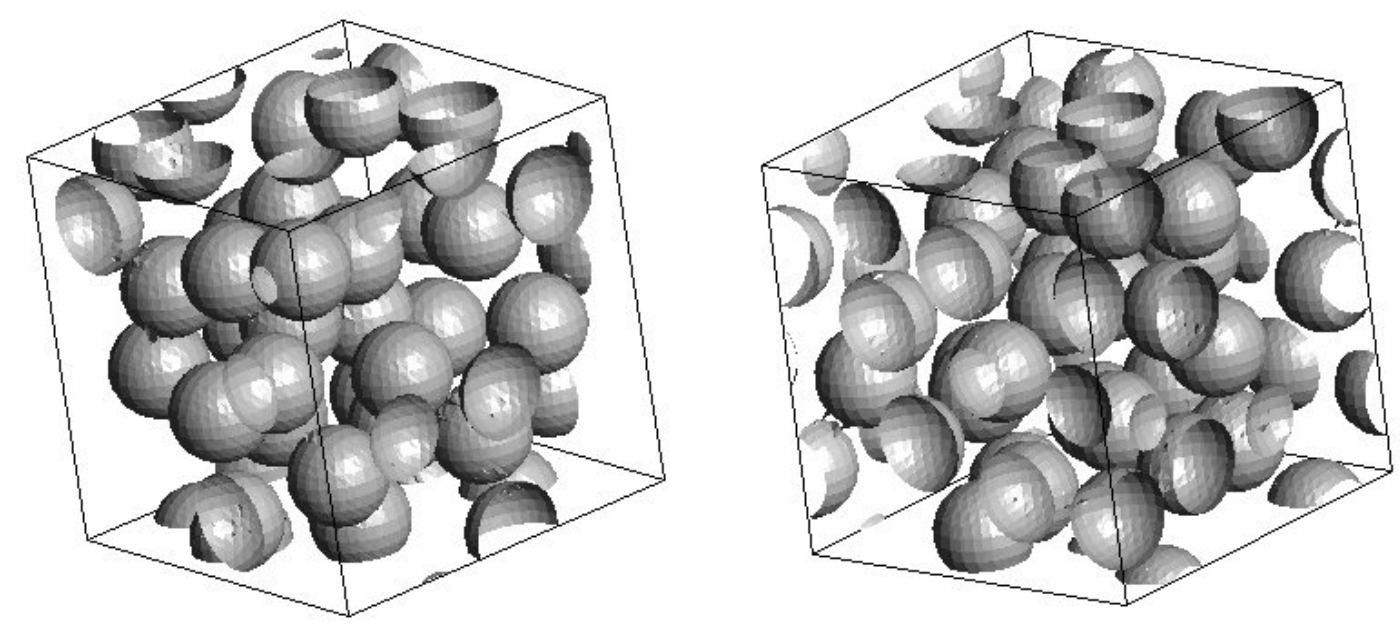

Fig. 16. Two different draws with 32 particles. 

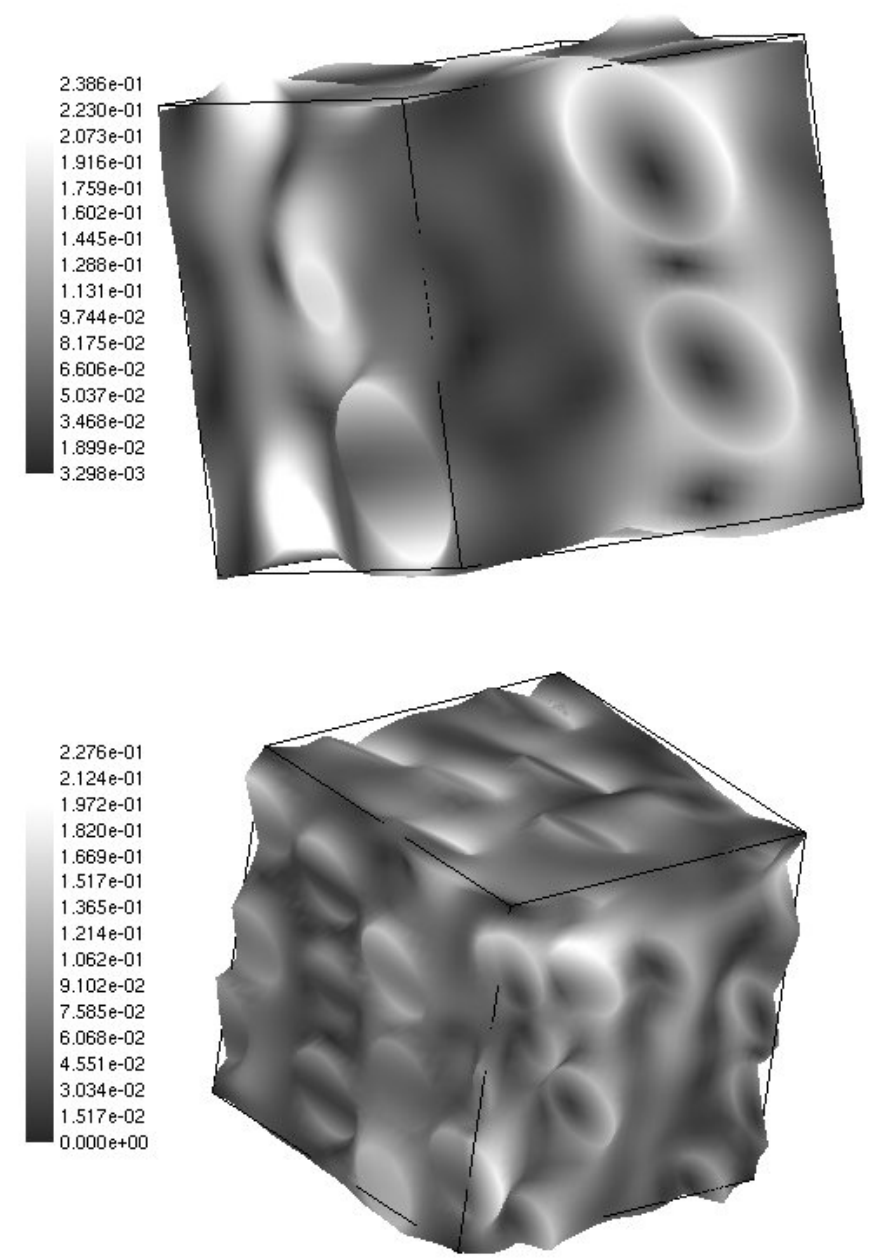

Fig. 17. Shear $x y$ deformation modes obtained by the X-FEM for two cells with 8 spheres (top) and 32 spheres (bottom).

\section{CONCLUSIONS}

The paper has demonstrated the applicability of the X-FEM to the homogenization of periodic basic cells. Since the mesh does not need to conform to the material interfaces, complex and random cell geometries can easily be computed with very limited meshing effort. The results obtained are very close to direct FEM and Fast Fourier Transform approaches. Moreover the X-FEM is also applicable to cells containing voids and phases with nonlinear constitutive laws. However, in order to reasonably locate the interface, the mesh must not be too coarse. A simple geometrical adaptivity scheme has been proposed. Finally, a new enrichment to introduce discontinuous strains inside an element has been developped. The initial results are promising since this enrichment provides a very close convergence rate to the "optimal" FEM convergence rate. 


\section{References}

[1] T. Belytschko and T. Black. Elastic Crack Growth in Finite Elements with Minimal Remeshing. International Journal for Numerical Methods in Engineering, 45(5), 601-620, 1999.

[2] T. Belytschko, C. Parimi, N. Moës, N. Sukumar and S. Usui. Structured extended finite element methods of solids defined by implicit surfaces. International Journal for Numerical Methods in Engineering, 56, 609-635, 2003.

[3] A. Bensoussan, J.L. Lions, G. Papanicolaou Asymptotic Analysis for Periodic Structures. North-Holland, Amsterdam, 1978.

[4] H.J. Bőhm. A short introduction to basic aspects of continuum micromechanics. Cdl-fmd Report 3-1998, http://ilfb.tuwien.ac.at/links/mom_m.html,TU Wien, Vienna, 1998.

[5] P.W. Chung, K.K. Tamma and R.R. Namburu. Asymptotic expansion homogenization for heterogeneous media: computational issues and applications. Composites: Part A 32, 1291-1301, 2001.

[6] C. Daux, N. Moës, J. Dolbow, N. Sukumar and T. Belytschko. Arbitrary branched and intersecting cracks with the eXtended Finite Element Method International Journal for Numerical Methods in Engineering, 48, 1741-1760, 2000 .

[7] S. Ghosh, K. Lee, and S. Moorthy. Multiple scale analysis of heterogeneous elastic structures using homogenization theory and Voronoi cell finite element method. Int. J. Solids Struct., 32(1),27-62, 1995.

[8] J.M. Guedes, and N. Kikuchi. Preprocessing and postprocessing for materials based on the homogenization method with adaptive finite element methods.Comp. Meth. in Applied Mech. and Engrg. 83, 143-198, 1990.

[9] S.J. Hollister and N. Kikuchi. Homogenization theory and digital imaging: a basis for studying the mechanics and design principles of bone tissue. Biotech. Bioeng. 94 43, 586-596, 1994.

[10] M. Kamiński. Boundary element method homogenization of the periodic linear elastic fiber composites. Eng. Anal. with Boundary Elements 23(10), 815-823, 1999.

[11] J.M. Melenk and I. Babuška. The Partition of Unity Finite Element Method: Basic theory and applications. Comp. Meth. in Applied Mech. and Engrg. 39, 289-314, 1996.

[12] J.C. Michel, H. Moulinec, and P. Suquet. Effective properties of composite materials with periodic microstruture: a computational approach. Comput. Meth. Appl. Mech. Eng. 172(1-4), 109-143, 1999. 
[13] J.C. Michel, H. Moulinec and P. Suquet. Homogénéisation en mécanique des matériaux 1, Chapter Composites microstructure périodique, pp. 57-94, Hermes Science, 2001.

[14] N. Moës, J. Dolbow and T. Belytschko. A finite element method for crack growth without remeshing. International Journal for Numerical Methods in Engineering 46, 131-150, 1999.

[15] N. Moës, A. Gravouil and T. Belytschko. Non-planar 3D crack growth by the extended finite element and level sets.Part I: Mechanical model. textitInternational Journal for Numerical Methods in Engineering. 53, 2549$2568,2001$.

[16] N. Moës, J. T. Oden and T. I. Zhodi. Investigation of the interactions between the numerical and the modeling errors in the Homogenized Dirichlet Projection Method. Comp. Meth. in Applied Mech. and Engrg. 159, 79-101, 1998.

[17] H. Moulinec and P. Suquet. A numerical method for computing the overall response of nonlinear composites with complex microstructure.Comput. Meth. Appl. Mech. Eng. 157, 69-94, 1997.

[18] H. Okada, Y. Fukui and N. Kumazawa. Homogenization method for heterogeneous material based on boundary element method. Computers \&3 Structures 79(20-21), 1987-2007, 2001.

[19] J.-F. Remacle and C. Geuzaine. Gmsh finite element grid generator. Available at www.geuz.org/gmsh, 1998.

[20] J.-F. Remacle, B. Kaan Karamete and M.S.Shephard. Algorithm Oriented Mesh Database.In Ninth International Meshing Roundtable. 2000.

[21] J.-F. Remacle, B. Kaan Karamete and M.S. Shephard. AOMD: Algorithm Oriented Mesh Database.Available at http://www.scorec.rpi.edu/AOMD. 2001.

[22] E. Sanchez-Palencia. Non homogeneous media and vibration theory, Volume 127 of Lecture Notes in Physics. Springer Verlag, Berlin, 1980.

[23] J. A. Sethian.Level Set Methods \& Fast Marching Methods:Evolving Interfaces in Computational Geometry, Fluid Mechanics, Computer Vision, and Materials Science. Cambridge, UK: Cambbridge University Press. 1999.

[24] N. Sukumar, D. L. Chopp, N. Moës and T. Belytschko. Modeling Holes and Inclusions by Level Sets in the Extended Finite Element Method. Comp. Meth. in Applied Mech. and Engrg. 190, 6183-6200, 2001.

[25] P. Suquet. Elements of homogenization for inelastic solid mechanics. In E. Sanchez-Palencia and A. Zaoui (Eds.), Homogenization Techniques for Composite Media, Volume 272 of Lecture Notes in Physics, pp. 193-278. Springer Verlag, Berlin, 1985.

[26] R. Wentorf, R.Collar, M.S. Shepard and J. Fish. Automated modeling for complex woven microstructures. Comput. Meth. Appl. Mech. Eng. 172(1-4), 273-291, 1999. 
[27] T. Zohdi, M. Feucht, D. Gross and P. Wriggers. A description of macroscopic damage through microstructural relaxation. Int. J. Num. Meth. Eng., 143, 493506, 1998. 